\title{
Argumentaire sur la nécessaire souveraineté du Québec
}

\author{
Mélodie Caron
}

Université Condordia

Diplômée de l'Université Concordia en science politique au printemps 2007, Mélodie Caron vécut quatre années à dédier efforts et passions à l'étude des théories et des systèmes politiques d'ici et d'ailleurs. Bien que son attention fût généralement tournée vers la politique comparée de l’Asie du Sud et du Nord-Est, ainsi que la géopolitique et la religio-politique du Moyen Orient, la politique québécoise et canadienne fut toujours sujet à soulever ses ardeurs. Étant partisane du mouvement souverainiste québécois, elle s'impliqua avec le Conseil de la Souveraineté du Québec et compte bien dédier temps et compétences à la promotion des orientations souverainistes dans les années suivant sa graduation. 


\section{Préface}

Pour ne laisser aucune place à la subtilité ou l'ambiguïté, il semble important de positionner l'orientation de cet ouvrage dès les premières lignes. Le texte argumentatif qui suit est de nature souverainiste. Il s'emploie à développer un argumentaire construit autour d'une thèse très simple; le Québec, de par son identité distincte, a les moyens de pallier à la déficience du fédéralisme canadien et de s'affranchir du statut de province fédérée. De plus, il est important de préciser que la structure de cet ouvrage ne répond pas de l'approche cartésienne de la thèse, antithèse, synthèse valorisée par les milieux académiques. La raison en est bien simple, la thèse de cet essai naît de la vision québécoise de l'histoire canadienne, c'est-à-dire que l'état canadien est né d'une entente entre deux nations, francophone et anglophone. Se positionnant comme tel, la thèse ne cherche pas a débattre ou a exposer d'autre vision historique de la fédération canadienne, croyant donc superflu de discuter de la vision prédominante dans le canada anglais qui perçoit la création de la nation canadienne comme un moyen d'unisson contre le pouvoir colonial britannique. Dela même façon, si l'argumentaire s'appuie sur une vision de la fédération canadienne centralisatrice des pouvoirs à Ottawa, l'ouvrage ne sent pas le besoin de décrire l'opinion opposée, généralement embrassée par les fédéralistes, qui perçoit la fédération comme une forme de pouvoir politique décentralisé. L'intention avoué d'ignorer les contrearguments ou les visions politiques opposées provenant du Canada anglais répond a la volonté première de l'ouvrage qui est d'exposer l'argumentaire souverainiste et ainsi renforcir l'argument d'appartenance nationale du peuple québécois.

\section{Introduction}

Depuis près d'un demi-siècle, et de façon plus prononcée depuis les modifications constitutionnelles de 1982, le fédéralisme canadien a pris la tangente d'une centralisation toujours plus grande. Les politiques du gouvernement central, qu'elles concernent le pouvoir fédéral de dépenser, la Charte des droits et libertés canadiennes ou les lois du bilinguisme et du multiculturalisme, ne correspondent plus à la nature même du fédéralisme canadien et s'éloignent de façon irréversible d'un « accommodement raisonnable » avec le peuple québécois. La marginalisation de la majorité francophone du Québec, objectif non avoué des politiques multiculturalistes et centralisatrices d'Ottawa, a favorisé et favorise toujours les sentiments à 
la fois d'émancipation et d'exclusion qui sont à l'origine du mouvement souverainiste au Québec. Bien que le nationalisme québécois moderne en soit un d'affirmation positive et, parallèlement, de réaction contre le projet de nation unitaire du Canada, c'est pourtant le sentiment d'appartenance identitaire distinct des Québécois qui est le réel agent nourricier des volontés souverainistes de ce peuple francophone d'Amérique du Nord.

\section{L'aliénation du Québec dans la fédération canadienne}

«Qu'ils se soient appelés Duplessis, Sauvé ou Lesage, nos chefs d'État se sont tous heurtés rapidement aux barrières qu'impose à notre évolution le gouvernement central, par une habile exploitation des obscurités de l'Acte de l'Amérique du Nord britannique.»

- Pierre Bourgault - 1961

Lorsque le Canada décida de s'affranchir de la tutelle colonisatrice en 1867, le pacte était bien clair; le Canada était une entente entre deux peuples fondateurs, anglophones et francophones. C'est cette composition dualiste des peuples canadiens qui poussèrent les « Pères de la Confédération » à opter pour une

\footnotetext{
${ }^{1}$ En ligne. http://quebeclibre.net/bourgault.html. Consulté le 20 novembre 2006
}

structure politique de type fédéraliste. Comme le veut la définition classique d'une fédération, l'allégeance à un concept étatique dit fédéré impliquait alors la création de deux paliers de gouvernement où chacun exerçait sa souveraineté dans le cadre de ses champs de compétences respectifs. Pour le peuple fondateur francophone, le fédéralisme représentait un outil devant lui assurer l'autonomie politique dans ses sphères de responsabilités attribuées par les textes constitutionnels, ainsi que lui donner les moyens des mesures nécessaires à la préservation de son identité propre. ${ }^{2}$ Il est même un fait commun d'entendre que la Confédération de 1867 fut en fait un pacte de reconnaissance de la spécificité québécoise, pour sa langue, ses origines, mais surtout pour la reconnaissance de sa culture. La fédération canadienne existe donc grâce, ou à cause du Québec, car sans la présence de ce peuple de culture distincte, le Canada serait certainement entré dans le rang des États unitaires communément appelés État Nation. ${ }^{3}$

Suivant la logique initiale de la fédération canadienne, il semble plutôt légitime que les Québécois, toujours majoritairement francophones, cherchent à

\footnotetext{
${ }^{2}$ Facal, Joseph. Le déclin du fédéralisme canadien. P.17

${ }^{3}$ Jean H. Guay \& François Rocher. De la difficile reconnaissance de la spécificité québécoise. p. 61
} 
préserver la caractéristique décentralisée de l'État canadien. Ce l'est d'autant plus que la nature de l'identité québécoise a vécu de grands changements au cours du 20 ème siècle. La Révolution tranquille des années 60 fut l'éveil du peuple québécois. Elle représente la rupture avec l'identité canadienne-française de peuple fondateur pour adopter une identité culturelle et linguistique propre aux Québécois. La spécificité québécoise est dès lors plus qu'une simple question de protection linguistique ou de distinction religieuse, mais plutôt une volonté d'autodétermination politique dans les champs de compétences fondamentaux à la survie de cette culture, nommément l'éducation, la santé, les services sociaux et l'économie. ${ }^{4}$ Dans les mots de J ean H. Guay et François Rocher, auteurs de l'essai Dela difficile reconnaissance de la spécificité québécoise, au sortir de la Révolution tranquille: « le nationalisme québécois n'en est plus un de défense mais de croissance $» .5$

Il n'est donc pas surprenant de voir la province de Québec se battre avec acharnement pour la préservation du type de fédéralisme à son état initial. Ses volontés à cet égard sont bien simples : Elles consistent en l'acceptation du dualisme linguistique et culturel qui caractérise le pays, ainsi qu'en le respect de

\footnotetext{
${ }^{4}$ Jean H. Guay \& François Rocher, Loc. Cit., p. 65

${ }^{5}$ Ibid, p. 66
}

l'autonomie politique et institutionnelle des provinces qui passent, bien sûr, par le respect des compétences et des responsabilités exclusives de ces dernières. ${ }^{6}$

Malheureusement, bien que simples, ces volontés n'ont pu être respectées par l'autorité centrale. Plutôt que de chercher à préserver la composante essentielle à la survie de la fédération, c'est-à-dire la décentralisation des pouvoirs, Ottawa s'affaire à de grands projets de centralisation des politiques d'État qui ont pour effet direct la marginalisation du peuple québécois. Cette tendance du gouvernement central s'exprime, entre autres, par le pouvoir fédéral de dépenser. Une pratique qui prend racine dans les années 1940 lors de la Seconde Guerre mondiale. À cette époque les provinces, pour participer à l'effort de guerre, ont volontiers partagé leur assiette fiscale avec l'autorité centrale. Au sortir de la guerre, le gouvernement, plutôt que d'accorder un retour complet à leurs capacités fiscales, retourna aux provinces une redevance en espèce tout en imposant lui-même les règles des programmes à frais partagés. Ottawa s'immisce alors dans une multitude de programmes sociaux comme par exemple l'assurance hospitalisation en 1957, l'assurance maladie et la sécurité du

\footnotetext{
${ }^{6}$ Facal, Joseph, Loc. Cit., p. 54
} 
revenu en 1966 ainsi que le financement de l'enseignement postsecondaire en $1967 .{ }^{7}$

Le pouvoir fédéral de dépenser est certainement un sujet de division au sein des provinces canadiennes. Au Québec, cette tendance de l'autorité centrale de faire des « cadeaux » en argent dans les champs de compétences exclusifs aux provinces est perçu comme une entrave à l'autorité provinciale et un non respect de la constitution. Au Québec, toujours, le pouvoir fédéral de dépenser se définit comme « un instrument de centralisation (...) réussissant l'un des effets de légitimation idéologique les plus spectaculaires du vocabulaire constitutionnel (...) ». ${ }^{8} \mathrm{En}$ d'autres mots, le pouvoir fédéral de dépenser n'est rien d'autre qu'une affirmation idéologique d'un pouvoir inexistant selon les textes constitutionnels.

\section{La bataille du Québec contre le Pouvoir Fédéral de dépenser}

Bien sûr, la question est épineuse. N'estce pas délicat de reprocher à son gouvernement de dépenser l'argent perçu par les impôts et les taxes? Dépenser l'argent de la collectivité à bon escient, n'est-il pas là la fonction première de tout bon gouvernement? En fait, voici où le bât

\footnotetext{
${ }^{7}$ Facal, Joseph, Loc. Cit., p.24

${ }^{8}$ Commission sur le déséquilibre fiscal; Le «pouvoir fédéral de dépenser». p.8-9
}

blesse. Dans un système d'État fédéré comme celui du Canada, où chaque palier de gouvernement détient ses champs de compétences et d'autorités respectifs, le simple fait de percevoir de l'argent par les taxes et les impôts n'autorisent en rien la liberté complète d'en disposer. 9

\section{L'injection d'argent par le fédéral dans} les zones de compétences provinciales a un résultat palpable : « l'étranglement des provinces ${ }^{10}$. Depuis les années 1990, les politiques d'élimination de la dette n'ont fait qu'accélérer ce processus d'appauvrissement fiscal des provinces et du Québec en particulier. Par exemple, le fédéral s'est appropriéles surplus de la caisse d'assurance-emploi, surplus créé sur le dos des provinces et du monde du travail par l'augmentation des cotisations et la diminution de l'accessibilité qui entraîna une déviation de la clientèle en besoin vers les services sociaux, qui eux relèvent de la compétence des provinces. ${ }^{11} \mathrm{Il} \mathrm{a}$ également considérablement diminué les transferts financiers aux provinces sans pour autant alléger le fardeau fiscal des contribuables. En plus d'éliminer son déficit, ces politiques donnèrent lieu à d'agréables surplus budgétaires que le gouvernement

\footnotetext{
${ }^{9}$ Commission sur le déséquilibre fiscal, Loc. Cit., p. 12

${ }^{10}$ Facal, Joseph, Loc. Cit., p. 22

${ }^{11}$ Ibid, p26-27
} 
fédéral s'affaire à dépenser comme bon lui semble, entre autres dans les zones d'activités exclusives aux provinces. ${ }^{12}$

Suivant un tollé de protestation de la part du gouvernement du Québec, le gouvernement central s'était engagé, dans son Discours du Trône de février 1996, à mettre de côté son pouvoir de dépenser pour la création de programmes à frais partagés dans les champs de compétences provinciales s'il n'obtenait pas l'appui majoritaire des provinces. Pourtant, un an plus tard, ce même gouvernement informe le peuple canadien de ses projets unilatéraux dans les domaines de l'éducation, la santé et les services sociaux, tous de compétences exclusives aux provinces et fondamentales à la société distincte qu'est le Québec.13 Lucien Bouchard, alors Premier Ministre du Québec, soutient une position ferme face à l'autorité fédérale :

Sil est une chose au Québec, c'est bien le respect des compétences exclusives du Québec et, plus particulièrement, en matière de santé, de sécurité du revenu, de l'éducation, de politique familiale. Depuis trente ans, le gouvernement fédéral cherche à simmiscer dans les compétences du Québec. Il le fait essentiellement par le biais de ce qu'on appelle un pouvoir dit de dépenser que le Québec n’a jamais

\footnotetext{
${ }^{12}$ Facal, Joseph, Loc. Cit., p. 20

${ }^{13}$ Position historique du Québec sur le pouvoir fédéral de dépenser 1944-1998. p. 31
}

voulu reconnaître, cherchant à protéger ses compétences exclusives. (... $)^{14}$

\section{Ce discours de Lucien Bouchard,} prononcé en 1998, sonne quelque peu répétitif dans l'historique québécois de cette lutte contre le pouvoir fédéral de dépenser. En effet, la position du Québec en ce sens n'a jamais sourcillé depuis le gouvernement de Maurice Duplessis en 1944. À cette époque, le gouvernement Duplessis se battait pour que l'autorité fédérale lâche prise sur certains revenus de taxe pour ainsi permettre aux provinces de combler le manquement fiscal à l'exercice de leurs pleines compétences sans dépendre continuellement des subventions fédérales. ${ }^{15}$ C'est d'ailleurs suite à cette bataille infructueuse, et au besoin urgent du Québec de pallier à son manquement fiscal, que le gouvernement de M. Maurice Duplessis adopta, en 1954, une loi créant un impôt provincial sur le revenu des particuliers. ${ }^{16}$

\section{C'est le gouvernement de M. J ean} Lesage (1960-1966) qui obtiendra le plus de gains en matière de lutte contre l'ingérence du fédéral dans les champs de compétences

\footnotetext{
${ }^{14}$ Notes pour une allocution de M. Lucien Bouchard, à l'occasion d'une séance de la commission des institutions portant sur la Déclaration de Calgary, le 10 juin 1998, p. 7-8 (Position historique de Québec sur le pouvoir fédéral de dépenser. 1944-1998. p. 32 )

${ }^{15}$ Position historique du Québec sur le pouvoir fédéral de dépenser 1944-1998, Loc. Cit., p. 3

${ }^{16}$ Ibid. p.5
} 
provinciaux. Lors de son discours d'ouverture à la Conférence fédérale-provinciale de juillet

1960 à Ottawa, M. Lesage s'attaque

vigoureusement à la déficience des

programmes à frais partagés et aux subventions conditionnelles qu'Ottawa accorde aux provinces :

L'expérience démontre que souvent ces programmes conjoints ne permettent pas aux provinces d'utiliser leurs propres revenus comme elles l'entendent et de tenir suffisamment compte des conditions locales. De plus, ils soulèvent aussi des difficultés administratives qui signifient perte d'efficacité ou double emploi et des frais plus élevés. Les provinces doivent avoir à leur service un personnel spécialement chargé de faire rapport à Ottawa de l'exécution de ces programmes et le gouvernement fédéral doit à son tour engager des fonctionnaires pour voir à ce que les conditions exigées par Ottawa soient remplies par les provinces. ${ }^{17}$

C'est à la suite de ce discours et de cette conférence que le Québec obtiendra un droit de retrait des programmes à frais partagés moyennant une compensation financière sous forme de points d'impôt ou de versements de péréquation. Le gouvernement de J ean Lesage se retire donc des programmes conjoints déjà actifs et prend l'engagement de ne plus

\footnotetext{
${ }^{17}$ Discourt d'ouverture de M. Jean Lesage, Conférence fédérale-provinciale, Ottawa, 25-27 juillet 1960, p. 35. (Position historique du Québec sur le pouvoir fédéral de dépenser 1944-1998. p. 8)
}

participer aux programmes de ce type dans le futur. ${ }^{18}$ Cette pratique appelée « opting out » permettait, en théorie, l'implantation de relations fédérales-provinciales plus respectueuses de l'autonomie du Québec dans ses zones de compétence.

Par contre, ce droit de retrait n'élimine pas complètement l'ingérence du fédéral dans les zones de responsabilités exclusives à Québec. Par exemple, en ce qui à trait à la loi fédérale sur l'assurance-maladie, le gouvernement du Québec s'est retiré de ce programme à frais partagés en vertu de son droit de retrait pour implanter un programme complètement géré par Québec. Le gouvernement central contraignit tout de même Québec à demeurer dans les cadres d'un programme d'assurance-maladie équivalant et respectant les règles fixées par Ottawa même si le domaine de la santé est un champ de compétence exclusif aux provinces. ${ }^{19}$

Au fils des ans, ce droit au « opting out » fut lentement relégué aux oubliettes, dû en grande partie à l'essoufflement des gouvernements successifs du Québec devant l'opposition d'Ottawa au statut spécial du Québec en ce qui a trait aux programmes

\footnotetext{
${ }^{18}$ Position historique du Québec sur le pouvoir fédéral de dépenser 1944-1998, Loc. Cit., p.12

${ }^{19}$ Ibid. p.14
} 
conjoints. Les différents ministres fédéraux des finances, de 1964 à 1977 particulièrement, se sont succédé en ayant une volonté commune : « (...) la normalisation-déstabilisation du statut particulier que le Québec menaçait de développer de plus en plus dans les politiques sociales (...) »20. Les stratégies du fédéral, cherchant d'abord à banaliser les exigences de Québec, ont donné naissance à ce que l'on appellera « federal opting out », qui consiste à empêcher le Québec d'obtenir seul un droit de retrait, donc de donner à d'autres provinces ce auquel Québec a droit. ${ }^{21}$ Cette stratégie, répondant au besoin de diluer les intérêts $d u$ Québec pour la protection de sa distinction, reviendra de façon quasi régulière dans l'attitude d'Ottawa face aux demandes originales de Québec.

Comme nous l'avons abondamment illustré, l'ingérence récurrente du fédéral dans les responsabilités provinciales traduit une volonté d'homogénéisation des politiques de programmes sociaux à l'échelle du pays ainsi qu'une nécessaire présence et visibilité du fédéral au niveau de l'individu. ${ }^{22}$ Les secteurs les plus touchés par ce pouvoir fédéral de dépenser sont la santé, l'éducation, la famille,

\footnotetext{
${ }^{20}$ Yves Vaillancourt. Un bilan de l'opting out du Québec des programmes fédéraux à frais partagés dans le domaine social (1964-1992). p. 357

${ }^{21}$ Ibid. p. 361

${ }^{22}$ Facal, Joseph, Loc. Cit., p. 21
}

les démunis, la recherche et les nouvelles technologies. Il s'agit là de domaines stratégiques à l'implantation d'une uniformité pancanadienne à laquelle le Québec se devait et se doit toujours de réagir. L'exigence par Québec du respect des compétences exclusives par les deux paliers de gouvernement est un moyen de défense essentiel à la protection culturelle et identitaire de la société québécoise. L'agitation de Québec depuis plus de 60 ans a pour but de contrecarrer l'avancée de l’État fédéral canadien vers un système d'État unitaire. ${ }^{23}$

Un autre exemple éloquent de cette tendance unitaire s'observe dans l'ententecadre sur l'union sociale de février 1999 qui détermine les politiques sociales, champ de compétence exclusif des provinces selon les textes constitutionnels, comme devenant une zone de responsabilité partagée. Cette entente, qui n'est d'ailleurs pas un amendement à la constitution, fut signée sans Québec et en échange d'une hausse du financement fédéral en matière de programmes sociaux pour les autres provinces canadiennes. Elle permet donc à Ottawa de dicter des règles pancanadiennes, répondant à « l'identité canadienne », pour le financement des

\footnotetext{
${ }^{23}$ Facal, Joseph, Loc. Cit., p. 33-34
} 
programmes sociaux du pays. ${ }^{24}$ Il va sans dire que la volonté distincte de Québec dans l'élaboration de ses programmes sociaux devient ce que J oseph Facal, alors en poste au ministère des Affaires intergouvernementales canadiennes, appelle une « entrave à l'édification du nouveau Canada ».25 Cette volonté claire et avouée d'uniformiser les règles en matière de programmes sociaux fait $d u$ Canada un pays toujours plus centralisé, s'éloignant dangereusement de la nature même d'un État fédéraliste et reniant un peu plus chaque jour le contrat originalement signé avec l'autre peuple fondateur, les francophones.

\section{L'ère Trudeau et la Charte des droits et libertés}

Le pouvoir fédéral de dépenser, autant nuisible soit-il pour l'autodétermination de la province de Québec dans le cadre de ses sphères de compétences exclusives, n'est pas l'unique stratagème mis en place par Ottawa pour affaiblir le sentiment distinctif de la société québécoise. L'ère Trudeau fut en quelque sorte un couteau dans le cour pour tous les Québécois croyant encore à la survie, voire au bien être, de la nation québécoise au sein du fédéralisme canadien. Pierre Elliott Trudeau avait des visions bien particulières pour la nation canadienne. Il eut du moins la

\footnotetext{
${ }^{24}$ Facal, Joseph, Loc. Cit., p. 37

${ }^{25}$ Ibid. p. 38
}

franchise d'avouer la nature centralisée de la fédération :

Il n'existe pas, ce «bargaining
power». Il n'a jamais existé. A
preuve, en 52 ans, on n'a pas
rapatriéla Constitution, et il n'y a
pas eu plus de pouvoirs donnés
aux provinces. Au contraire, les
deux amendements, celui de 1940
et de 1953 [...] ont accordé plus de
pouvoirs au gouvernement
fédéral. ${ }^{26}$

Et il s’appliqua à solidifier cette logique de domination du gouvernement central en entamant les modifications à la constitution de 1982. Dans ces grandes lignes, la Loi constitutionnelle de 1982 touche cinq points principaux : La Charte des droits et libertés portant sur les libertés fondamentales, le droit démocratique, le droit à l'égalité, le droit à l'enseignement dans la langue de la minorité, les langues officielles/le bilinguisme ainsi que la constitutionnalisation des principes de péréquation. ${ }^{27}$

La Charte des droits et libertés, bien qu'il soit difficile d'en nier les vertus, est en fait le problème majeur pour l'intégrité sociale et politique du Québec des modifications constitutionnelles de 1982. Selon Guy Laforest, auteur de La Charte canadienne des droits et

\footnotetext{
${ }^{26}$ Facal, Joseph, Loc. Cit., p. 36

${ }^{27}$ François Rocher \& Daniel Salée. Logique d'État et fédéralisme canadien : l'improbable décentralisation. p. 100102
} 
libertés au Québec : nationaliste, injuste et illégitime, la Charte n'est ni plus ni moins qu'un outil « d'édification de la nation canadienne $» .28$

Dans un premier temps, la Charte va à l'encontre même de la nature du fédéralisme en accordant une place prépondérante au pouvoir judiciaire, symbole de centralisation vu la nomination unilatérale par le pouvoir fédéral des juges de la Cour suprême. En ce sens, la Charte nuit considérablement au Québec en privilégiant une approche dite atomiste où l'individu devient un sujet juridique, ce qui bloque toute possibilité des gouvernements provinciaux de diriger leurs politiques au nom des intérêts de leur collectivité. ${ }^{29}$ Ceci représente en fait un grand pas vers la « judiciarisation du système politique canadien $»$.

\section{Dans un deuxième temps, il émane de} cette Charte une volonté de centralisation au niveau de l'idéologie par la création d'une culture politique et sociale dite pancanadienne. En d'autres mots, la Charte canadienne des droits et libertés vient homogénéiser les politiques publiques et sociales d'un océan à l'autre, détruisant ainsi la diversité régionale

\footnotetext{
${ }^{28}$ Guy Laforest. La Charte canadienne des droits et libertés au Québec : nationaliste, injuste et illégitime. p. 131

${ }^{29}$ François Rocher \& Daniel Salée. Logique d'État et fédéralisme canadien : l'improbable décentralisation. p. 102
}

qui fut longtemps l'une des caractéristiques propres au Canada. ${ }^{30}$

\section{L'impact sur la possibilité de} préservation de l'intégrité sociale québécoise est en ce sens désastreux. La Charte cherche à affaiblir le sentiment d'appartenance régionale et territoriale ${ }^{31}$ pour ainsi faire naître un attachement à la nation canadienne. Bref, elle s'affaire à diluer l'importance de la distinction culturelle, identitaire et linguistique du Québec dans une mer de groupes tous plus différents les uns que les autres, mais pourtant tous unis par une identité commune, celle d'être Canadien. Voilà ce que Guy Laforest a défini comme étant le minoritarisme constitutionnel.

Il semble essentiel à ce point-ci de la discussion de se remémorer le contrat initial; celui à la base même de la fédération canadienne qui unissait deux peuples fondateurs et qui reconnaissait la spécificité des Québécois francophones. La Charte des droits et libertés est en quelque sort la rupture avouée de ce contrat. La fin des amours, le début des procédures de divorce. Guy Laforest verbalise cette métaphore de façon on ne peut plus éloquente :

Le projet d'édification d'une seule et unique nation canadienne met en péril le projet de promotion

\footnotetext{
${ }^{30}$ Guy Laforest, Loc. Cit., p. 133

${ }^{31}$ Ibid, p. 134
} 
d'une identité et d'une culture distinctes au Québec. La Charte s'emploieà «déterritorialiser» les identités «locales» et «régionales» au profit de la communauté nationale canadienne et des institutions centrales. 32

Pierre Elliott Trudeau aura réussi son pari. Ses croyances en l'existence d'une seule nation canadienne s'appuyant sur le « bilinguisme et le multiculturalisme ainsi que son attachement profond à l'égalité des provinces et à la primauté des droits individuels » 33 font maintenant vigoureusement partie de l'inconscient collectif des Canadiens et ont considérablement meurtri la reconnaissance collective de la province francophone dont le Québec a essentiellement besoin pour continuer à vivre à l'intérieur du Canada.

\section{Le mirage de Meech}

Tel que précédemment illustré, la Loi Constitutionnelle de 1982 fut un véritable blocage à l'exercice de l'autonomie politique auquel les gouvernements provinciaux ont droit. Ce fut la négation des initiatives provinciales pour allouer à l'État central seulement la tâche de répondre aux intérêts nationaux. ${ }^{34}$ En réponse à cet accroc historique, les modifications constitutionnelles

\footnotetext{
${ }^{32}$ Guy Laforest, Loc. Cit., p. 147

${ }^{33}$ Facal, Joseph, Loc. Cit., p. 55

${ }^{34}$ François Rocher \& Daniel Salée, Loc. Cit., p. 105
}

du Lac Meech de 1987 furent l'ultime effort pour revenir à des amours plus civilisés entre les deux paliers de gouvernance. Elles visaient principalement à faire signer la constitution canadienne rapatriée par Québec et selon les conditions de Québec. Sous les gouvernements de Brian Mulroney à Ottawa et de Robert Bourassa à Québec, Meech se voulait un retour à une interprétation constitutionnelle basée sur la réalité de dualisme linguistique du Canada ainsi que sur la distinction culturelle et sociale de la province de Québec. L'Accord visait également quelques changements d'ordre institutionnel tels la nomination des sénateurs et des juges de la Cour Suprême ainsi que l'encadrement du pouvoir fédéral de dépenser. ${ }^{35}$

\section{L'Accord du Lac Meech fut d'abord} perçu comme une volonté du gouvernement de M. Mulroney de mettre un terme définitif à la tendance lourde de centralisation des pouvoirs à Ottawa, tendance accentuée par l'époque Trudeau et la Loi Constitutionnelle de 1982. Cette perception venait certainement de l'étude des trois objectifs avoués des discussions. Tout d'abord, Meech se voulait un agent d'harmonisation politique entre Québec et Ottawa. L'Accord cherchait également à imposer le respect des responsabilités

\footnotetext{
${ }^{35}$ François Rocher \& Daniel Salée, Loc. Cit., p.111
} 
provinciales pour ainsi mettre un terme à l'ingérence fédérale en ce domaine, puis s'orientait vers une dépolitisation du régime de subventions fédérales. ${ }^{36}$ Par contre, une observation plus poussée des véritables intentions exprimées par ces discussions intergouvernementales nous démontre qu'il ne s'agissait pas là d'une volonté décentralisatrice mais plutôt d'un effort pour obtenir l'accord des provinces à une centralisation constante des pouvoirs à Ottawa. ${ }^{37}$

Bref, si adopté selon les conditions posées par Québec, conditions pourtant faibles aux yeux des souverainistes québécois, l'Accord du Lac Meech aurait restreint considérablement la possibilité de recourir à l'unilatéralisme dans la construction des règles et des programmes politiques à Ottawa. Il aurait également reconnu le caractère distinct du Québec et son autonomie nécessaire à la protection de sa majorité francophone. ${ }^{38} \mathrm{Il}$ aurait en fait renoué avec l'idée originelle de la fédération canadienne et réintroduit une atmosphère politique basée sur la réelle dualité de la constitution, c'est-à-dire deux peuples, deux langues, deux cultures. Mais comme ces belles espérances allaient à l'encontre de la tangente fédéraliste de l'heure, Meech est mort

\footnotetext{
${ }^{36}$ François Rocher \& Daniel Salée, Loc. Cit., p. 111

${ }^{37}$ Ibid, 115

${ }^{38}$ Ibid, p.112-113
}

avant même de voir lejour. Le statut de société distincte du Québec est et demeurera relégué à un élément de diversité parmi plus de quatorze dénombrés par la « clause Canada ».39

Comme mentionné précédemment, c'est le fantôme de Pierre Elliott Trudeau qui dictera l'attitude fédérale face au Québec tout au long des années 1990 jusqu'à aujourd'hui. Et ce, même au lendemain du référendum de 1995, où, plutôt que de comprendre le besoin urgent de changement exprimé par un vote massif des Québécois en faveur de l'indépendance, Ottawa a répondu par une nouvelle vague de centralisation des pouvoirs, limitant toujours plus l'autonomie de la province. Québec est donc puni pour avoir exprimé son désir d'autodétermination politique. Le gouvernement fédéral lui a réservé un traitement spécial qui s'exprime par des règles de transferts financiers désavantageuses, un blocage de ses activités internationales et la Loi C-20 sur la clarté référendaire qui, en plus d'ignorer les règles de démocratie universelle, propage des préjugés ignobles sur l'incapacité de gouvernance démocratique des Québécois. ${ }^{40}$

Voilà donc ce qu'est devenu le Québec dans la fédération canadienne. Voilà surtout ce qui alimente le mécontentement québécois et

\footnotetext{
${ }^{39}$ Guy Laforest, Loc. Cit., p. 137

${ }^{40}$ Facal, Joseph, Loc. Cit., p. 23
} 
par le fait même la fougue indépendantiste. Et s'il n'y a qu'un seul point qui unit mon opinion à celle de J ohn Ralston Saul ${ }^{41}$, c'est que oui le nationalisme québécois en est un de réaction et d'opposition, un « nationalisme négatif » selon Saul, car dans le respect réel de ses différences, dans un système permettant au peuple québécois d'agir entant que nation reconnue, et de défendre et préserver ses distinctions culturelles et linguistiques, le Québec pourrait vivre à l'intérieur d'une fédération canadienne. Mais comme la reconnaissance n'existe plus et que la fédération l'est de moins en moins, rien de tout ça ne sera donc possible. En résumé, l'opinion souverainiste que je tente d'exprimer ici n'en est pas une de souveraineté à tout prix, mais bien de souveraineté si nécessaire, et je crois avoir démontré jusqu'à maintenant qu'il est politiquement essentiel pour la survie du Québec d'atteindre cet objectif. Maintenant, comment l'identité culturelle et linguistique du Québec est-elle une force suffisante à la réalisation de ce projet?

\section{L'argumentaire identitaire des souverainistes du Québec}

« Pour un petit peuple commele nôtre, sa situation minoritaire sur un continent anglo-saxon crée déjà une tentation permanente de ce refus de soi-même, qui a les

\footnotetext{
${ }^{41}$ John Ralston Saul. Reflection of a Siamese Twin; Canada at the Beginning of the Twentieth Century.
}

attraits d'une pente facile, au bas de laquelle se trouverait la noyade confortable dans le grand tout.»

- René Lévesque - $1968^{42}$

À la veille du référendum de 1995, un organisme informel appelé Les Intellectuels pour la souveraineté fut formé dans le but précis de développer un argumentaire répondant principalement à l'aspect identitaire de la volonté souverainiste du Québec. Ce groupe venait également répondre aux critiques de la classe politique du Québec déplorant l'inaction publique des intellectuels québécois dans la cause souverainiste. Il en résulta deux publications ayant pour titre Manifeste des intellectuels pour la souveraineté; Douze essais sur l'avenir du Québec paru en 1995 et Redonner un sens à la souveraineté paru dix ans plus tard, en 2005. Dans le but de déterminer comment l'identité culturelle et linguistique du Québec est une force suffisante à l'atteinte de la souveraineté. Je m’appuierai sur ces deux ouvrages d'importance pour le mouvement souverainiste des dix dernières années.

\section{L'identité culturelle}

L'identité culturelle, pilier central à tout sentiment nationaliste, s'exprime spontanément à travers l'histoire, l'art, les

\footnotetext{
${ }^{42}$ En ligne. http://quebeclibre.net/levesques.html. Consulté le 20 novembre 2006
} 
médias, etc. et permet à l'individu de s'attacher émotionnellement à une communauté plus large et diversifiée que ses simples liens familiaux. Plus encore, l'identité culturelle représente les fondements de l'inconscient collectif d'une communauté. Elle est « une construction symbolique qui trouve en partie ses racines dans la façon dont nous est conté ce que nous n'avons pas vécu directement $\gg .43$ Alors, si la nation est une « communauté imaginée ${ }^{44}$, le nationalisme en est le sentiment découlant, le symbole de l'imaginaire collectif.

L'argument identitaire pour la souveraineté du Québec trouve son essence dans cette définition del'identité culturelle. Comme démontré précédemment, le Québec s'est efforcé de vivre dans la fédération canadienne depuis maintenant près de 140 ans. A cette époque, il joignit les forces de son peuple aux forces d'un autre peuple pour la formation d'un État fédéré. Malheureusement, le Québec ne peut aujourd'hui souhaiter autre chose que la souveraineté vu le refus du Canada à préserver cette entente initiale.

C'est donc au tournant de la Révolution tranquille que le Québec embrasse un projet

\footnotetext{
${ }^{43}$ Marcos Ancelovici \& Francis Dupuis-Déri. L’Archipel identitaire; Recueil d'entretiens sur l'identité culturelle. p. 16 44 Terme régulièrement utilisé par Benedict Anderson.
}

national le dissociant de l'entité canadienne. S'orientant d'abord vers une recherche d'égalité entre Canadiens et Québécois, le projet national s'affirme finalement en tant que réel projet souverainiste sous le gouvernement de M. J ean Lesage; « la nécessité de préserver le caractère traditionnel du Canada français cédait le pas au besoin d'affirmation nationale (...) jugé indispensable à la tâche de modernisation à laquelle s'attelait l'État québécois ».45 La dualité nationale associée au Canada n'est dès lors plus apparentée à l'ethnicité de ses citoyens mais plutôt à la nature de leur culture. Daniel J ohnson décrit alors le Québec en tant que « communauté humaine manifestant une unité historique, linguistique, religieuse et économique animé d'un vouloir- vivre commun.»46 Et c'est surtout ce vouloir-vivre commun qui caractérise le nationalisme québécois depuis les années 1960.

Le nationalisme québécois hérité de la Révolution tranquille est le produit de deux définitions de sa communauté. Selon J acques Beauchemin, la nation québécoise est d'abord une communauté « imaginée », c'est-à-dire que les Québécois sont héritiers de leur destin, et que malgré la réalité multiculturelle du Québec moderne, et l'apport ethnique engendré par les

\footnotetext{
${ }^{45}$ François Rocher \& Michel Sarra-Bournet. Manifeste des intellectuels pour la souveraineté; Douze essais sur l'avenir du Québec : La longue quête de l'égalité. p. 50

${ }^{46}$ Idem.
} 
communautés immigrantes, le mouvement souverainiste ne doit pas renier son l'histoire. La nation québécoise est également une communauté « politique», c'est-à-dire que les Québécois sont citoyens d'une nation à part entière qui se doit d'être reconnue et respectée dans son intégrité. ${ }^{47}$ En d'autres mots, si l'argumentaire souverainiste du XXIème siècle doit embrasser la réalité pluraliste de sa société ainsi que la beauté des valeurs « ethicopolitiques » qu'engendre ce pluralisme, s'il doit demeurer inclusif dans sa définition de communauté politique; la souveraineté du Québec doit pareillement s'associer à l'appartenance à l'histoire de son peuple fondateur. 48

Michel Seymour élabore cette même idée sous la forme de complexité identitaire où le nationalisme culturel rencontre le nationalisme civique..$^{49} \mathrm{Il}$ souligne que le nationalisme culturel prend racine dans le trajet historique de la nation québécois alors que le nationalisme civique est né des réalités pluralistes de la nation québécoise actuelle, essentiellement depuis le référendum de 1995. En ses mots :

\footnotetext{
${ }^{47}$ Jacques Beauchemin. Redonner un sens à l'indépendance : La souveraineté au nom de la mémoire. p. 34

${ }^{48}$ Ibid. p. 34-35

${ }^{49}$ Michel Seymour. Redonner un sens à l'indépendance : La politique de la complexité identitaire. p. 155
}

L'histoire de la majorité nationale est à la base de l'histoire publique commune, qui concerne l'histoire de nos institutions publiques communes, mais cela est compatible avec des institutions publiques minoritaires qui complètent l'histoire des Québécois francophones. ${ }^{50}$

Le nationalisme québécois n'est donc plus que culturel mais bien civique, ce qui donna naissance à un mouvement souverainiste inclusif où la diversité culturelle est en fait la reconnaissance des identités multiples qui crée le dynamisme de la société québécoise.

\section{L'identité linguistique}

L'argument identitaire nous force nécessairement à discuter de l'argumentaire linguistique au sein du mouvement souverainiste, la langue étant un définitif primaire de l'identité culturelle. De façon simple et concise, la souveraineté du Québec n'est ni plus ni moins qu'un outil de protection et de promotion de la langue française en Amérique du Nord. Comme démontré plus haut, la Loi sur les langues officielles et le bilinguisme de Pierre Elliott Trudeau est venue renforcir la dualité francophone-anglophone au sein de la nation canadienne plutôt que de répondre à la nécessaire égalité linguistique exigée par les souverainistes.

\footnotetext{
${ }^{50}$ Michel Seymour, Loc. Cit., p. 156
} 
Dans un premier temps, le bilinguisme concerne spécialement et uniquement la fonction publique du gouvernement central qui, en tant que secteur majoritairement anglophone ouvrant ses portes à la minorité francophone, devient un véritable « foyer d'assimilation »51. Dans un deuxième temps, le bilinguisme n'est appliqué qu'au Québec c'està-dire que la Loi sur les langues officielles vient, de façon indirecte et non-officielle, exiger des Québécois qu'ils maîtrisent l'anglais pour un usage quotidien alors que rien de tel n'est exigé aux anglophones du Canada dans leur maîtrise du français. En d'autres mots, on s'attend pouvoir communiquer en anglais partout au Québec, alors qu'il est pratiquement impossible de communiquer en français dans le Canada anglais, mis à part au NouveauBrunswick. La Loi sur les langues officielles, qui est d'ailleurs venue contrecarrer la Charte de la langue française (Loi 101) qui faisait du français la langue officielle du Québec, n'est ni plus ni moins qu'un outil promotionnel de l'anglais au Québec, relayant aux oubliettes la cause francophone hors Québec et surexploitant de supposées violations des droits des anglophones au Québec.52 Il est aujourd'hui clair que la Loi sur les langues officielles, ou toutes les autres politiques fédérales, n'est pas parvenue à prôner le

\footnotetext{
${ }^{51}$ François Rocher \& Michel Sarra-Bournet, Loc. Cit., p. 52

${ }^{52}$ Ibid, p.53
}

développement du français au Canada. Pire encore, tous les efforts de francisation que le Québec tente de mettre en place depuis 1982 ne cesse de se confronter à l'idéologie du fédéral, c'est-à-dire le bilinguisme officiel et la négation de la nation québécoise. ${ }^{53} \mathrm{La}$ souveraineté est donc nécessaire au Québec pour lui permettre d'établir le français comme langue commune pour tous les Québécois, le français comme véritable langue nationale.

\section{La question de l'immigration}

De plus, en lien direct avec l'argumentaire linguistique et identitaire, la souveraineté devient nécessaire en ce qui concerne l'intégration des immigrants, car le Québec est, et est appelé à demeurer une société d'immigration. Dans la structure actuelle, les immigrants sont accueillis par la Fédération canadienne et choisissent le Québec comme l'une des dix provinces canadiennes. Ils y apprennent le français par obligation tout en étant conscient qu'il s'agit de la langue de la majorité au Québec, mais de la minorité au Canada, alors ils utilisent l'anglais, langue de la majorité canadienne, dans leur vie de tous les jours. ${ }^{54}$ Ce phénomène est d'autant plus

\footnotetext{
${ }^{53}$ Charles Castonguay \& Josée Legault. Manifeste des intellectuels pour la souveraineté; Douze essais sur l'avenir du Québec : Pour l'avenir du français. p. 101-106

${ }^{54}$ Guy Rocher. Manifeste des intellectuels pour la souveraineté; Douze essais sur l'avenir du Québec : Préface. p. 9
} 
difficile à freiner depuis la Loi constitutionnelle de 1982 par laquelle le Québec est devenu une province comme les autres. Dans les mots de Guy Rocher; « le caractère francophone du Québec paraît à bien des immigrants forcé et artificiel, dans un Canada qu'on leur a présenté comme étant bilingue et multiculturaliste ».55

\section{Les institutions pour la culture}

L'argument culturel, distinct de l'argument identitaire car fondé sur des nécessités institutionnelles, est également essentiel à l'argumentaire souverainiste. Ce dernier soutient la souveraineté pour la création d'un environnement propice au fleurissement et à la conservation de la culture québécoise. Comme elle le fait depuis près de 140 ans, mais de façon plus acharnée depuis la Révolution tranquille, la culture québécoise doit continuer de nourrir l'imaginaire collectif de son peuple. En ce sens la souveraineté viendrait créer un espace de liberté permettant à cette culture incroyablement riche et diversifiée de respirer profondément et de se laisser aimer de son peuple.

La culture au sens créatif et créateur est, tout comme la langue, un définitif d'identité. En ce sens, même dans un contexte fédéraliste, le Québec devrait être le seul responsable de

${ }^{55}$ Guy Rocher , Loc. Cit., p. 10 son enveloppe culturelle. Par contre, la vision du gouvernement central en est tout autre. Accorder à Québec le plein pouvoir politique de l'épanouissement de sa culture viendrait considérablement miner le projet d'identité canadienne commune d'un océan à l'autre tel que le prône le fédéral. ${ }^{56}$
Bien sûr, certains artistes, spécialement dans le milieu du cinéma, semblent bien heureux de profiter du dédoublement budgétaire résultant de l'existence de deux paliers de gouvernements. Certains croient même que cette situation contribue à créer de la compétition, et donc à diversifier les sources de financement qui font paraître l'enveloppe plus épaisse. ${ }^{57}$ Mathieu-Robert Sauvé et Geneviève Sicotte ${ }^{58}$ répondent à ces arguments que le Québec, dans la fédération canadienne, hypothèque considérablement son avenir culturel car le financement de la culture par Ottawa répond à un objectif bien précis; l'affaiblissement de la distinction québécoise au profit de l'uniformité canadienne. Bien que jamais cette culture forte ne s'estompera complètement, la souveraineté demeure un outil essentiel permettant « d’assurer le plein

\footnotetext{
${ }^{56}$ Mathieu-Robert Sauvé \& Geneviève Sicotte. Manifeste des intellectuels pour la souveraineté; Douze essais sur l'avenir du Québec : Entre l'art-porteur-de-drapeau et l'art-pour-l'art. p. 122

${ }^{57}$ Ibid, p. 124

${ }^{58}$ Ibid, p. $115-128$
} 
épanouissement de la culture dans un espace national stable et autonome».59

\section{La souveraineté; un projet solidaire}

Les argumentaires linguistique, identitaire et culturel nous amènent à comprendre que la souveraineté du Québec, c'est aussi une question de solidarité. L'État souverain que veut devenir le Québec est d'abord et avant tout un projet de société qui, en plus de désirer le maintien de sa stabilité sociétaire, recherche la légitimité politique. Contrairement au symbole abstrait qu'est le pouvoir fédéral pour les Québécois, le pouvoir politique du Québec représente la réalité, l'avenir, le pouvoir de changement. Ce projet de société qu'est la souveraineté veut rendre complet ce pouvoir qui est aujourd'hui limité par son statut de province.

De plus, s'ajoutant aux arguments de décentralisation pour mettre fin au doublage bureaucratique qu'imposent l'existence de deux paliers de gouvernements et d'égalité entre les deux communautés nationales fondatrices de l'État canadien, l'argumentaire constitutionnel est majeur et fondamental. Bien qu'abondamment discuté dans cet ouvrage, je crois essentiel de m'y attarder une dernière fois sous l'angle de la synthèse.

\footnotetext{
${ }^{59}$ Mathieu-Robert Sauvé \& Geneviève Sicotte, Loc. Cit., p.127
}

Depuis 1982, le Québec ne fait symboliquement plus partie de l'aspect constitutionnel du Canada. La Clause Canada limite dangereusement les pouvoirs de Québec en matière de législation linguistique, la Charte des droits et libertés, de par son poids juridique, favorise la centralisation des pouvoirs à Ottawa, et les discussions constitutionnelles du Lac Meech et de Charlottetown, cherchant à rapatrier le Québec dans la fédération, ont tous échoué. Le tableau des relations entre Québec et Ottawa est plutôt noir. En fait, il exprime l'impossibilité du tant espéré « accommodement raisonnable ». La volonté d'Ottawa de restreindre le Québec au statut de minorité parmi une mer de diversité, alors que Québec ne lâche pas prise sur son statut de nation distincte, traduit un profond sentiment de désaccord fondamental et irréconciliable.

\section{L'égalité citoyenne qui renie la} particularité de la collectivité québécoise, l'égalité culturelle qui banalise le français et les origines ethniques des francophones ainsi que l'égalité des provinces qui ignore le statut distinct du Québec ${ }^{60}$ sont autant d'expressions de l'impossible réconciliation entre la vision canadienne de la fédération et la vision

\footnotetext{
${ }^{60}$ François Rocher \& Michel Sarra-Bournet, Loc. Cit., p. 55
} 
Québécoise du respect de sa nation. Face à ce constat d'échec, on ne parle plus de souveraineté à tout prix mais bien de nécessaire souveraineté.

\section{Conclusion}

Il fut démontré tout au long de cet ouvrage que l'actuel contentieux politique entre le Québec et le Canada ne permet pas d'autre choix que la souveraineté. Cette souveraineté fut désirée et revendiquée depuis la Révolution tranquille, elle est devenu nécessaire depuis l'époque Trudeau vu le poids de son héritage dans l'inconscient collectif du Canada anglais. La souveraineté souhaitée par le peuple Québécois transcende l'arène politicoinstitutionnelle; elle s'exprime en termes d'identité, de langue et de culture, car les peuples, tout comme les individus, ont besoin d'être reconnus. La souveraineté représente cette légitime reconnaissance à la hauteur de sa spécificité, de sa beauté et de sa complexité. Elle devient un moyen de reconnaissance identitaire nationale et non une fin en soi. Dans les mots de Michel Seymour; « la souveraineté est une option politico-idéologique et non un déterminisme». ${ }^{61}$ Donc, loin de s'affaiblir, l'argumentaire souverainiste au Québec est plus pertinent que jamais.

${ }^{61}$ Michel Seymour, Loc. Cit., p. 162 


\section{Bibliographie}

ANCELOVICI, Marcos \& Francis DUPUIS-DÉRI. L'Archipel identitaire; Recueil d'entretiens sur l'identité culturelle. Édition Boréal, 1997, 214 p.

BEAUCHEMIN, J acques. Redonner un sens à l'indépendance. La souveraineté au nom de la mémoire. VLB éditeur, 2005, pp.19-35

CASTONGUAY, Charles \& J osée LEGAULT. Manifeste des intellectuels pour la souveraineté; Douze essais sur l'avenir du Québec. Pour l’avenir du français. Éditions Fides, 1995, pp.101-114

Commission sur le déséquilibre fiscal. Rapport : Annexe 2. Le « pouvoir fédéral de dépenser ». Dépôt légal; Bibliothèque nationale du Québec, 2002, ISBN : 2-550-38853-4.

FACAL, J oseph. Le déclin du fédéralisme canadien. VLB éditeur : Montréal, 2001, 70 p

GUAY, J ean H. \& François ROCHER. Bilan québécois du fédéralisme canadien. De la difficile reconnaissance de la spécificité québécoise. VLB éditeur : Montréal, 1992, pp.58-78

LAFOREST, Guy. Bilan québécois du Fédéralisme canadien. La Charte canadienne des droits et libertés au Québec : nationaliste, injuste et illégitime. VLB éditeur : Montréal, 1992, pp.124-151

LES INTELLECTUELS POUR LA SOUVERAINETÉ (IPSO). Manifeste des intellectuels pour la souveraineté; Douze essais sur l'avenir du Québec. Oui au changement. Éditions Fides, 1995, pp.15-21

Ministère du conseil exécutif. Secrétariat aux Affaires intergouvernementales canadiennes. Direction des politiques institutionnelles et constitutionnelles. Position historique du Québec sur le pouvoir fédéral de dépenser; 1944-1998. J uillet 1998, http:// www.cex.gouv.qc.ca/ saic/ position.htm

ROCHER, François \& Daniel SALÉE. Bilan québécois du Fédéralisme canadien. Logique d’État et fédéralisme canadien : l'improbable décentralisation. VLB éditeur : Montréal, 1992, pp.91-123

ROCHER, François \& Michel SARRA-BOURNET. Manifeste des intellectuels pour la souveraineté; Douze essais sur l'avenir du Québec. La longue quête de l'égalité. Éditions Fides, 1995, pp.43-57

ROCHER, Guy. Manifeste des intellectuels pour la souveraineté; Douze essais sur l'avenir du Québec. Préface. Éditions Fides, 1995, pp.7-12

SAUL, J ohn Ralston. Reflection of a Siamese Twin: Canada at the Beginning of the Twenty-First Century. Penguin Group : Toronto, 1997, 534 p.

SAUVÉ, Mathieu-Robert \& Geneviève SICOTTE. Manifeste des intellectuels pour la souveraineté; Douze essais sur l'avenir du Québec. Entre l'art-porteur-de-drapeau et l'art-pour-l'art. Éditions Fides, 1995, pp.115-128 
SEYMOUR, Michel. Redonner un sens à l'indépendance. La politique de la complexité identitaire. VLB éditeur, 2005, pp.153-176

VAILLANCOURT, Yves. Bilan québécois du Fédéralisme canadien. Un bilan de l'opting out du Québec des programmes fédéraux à frais partagés dans le domaine social (1964- 1992). VLB éditeur : Montréal, 1992, pp.345-371

VIENNE, Michel. Redonner un sens à l'indépendance. La souveraineté, une idée forte à l'heure dela mondialisation. VLB édition, 2005, pp.93-111

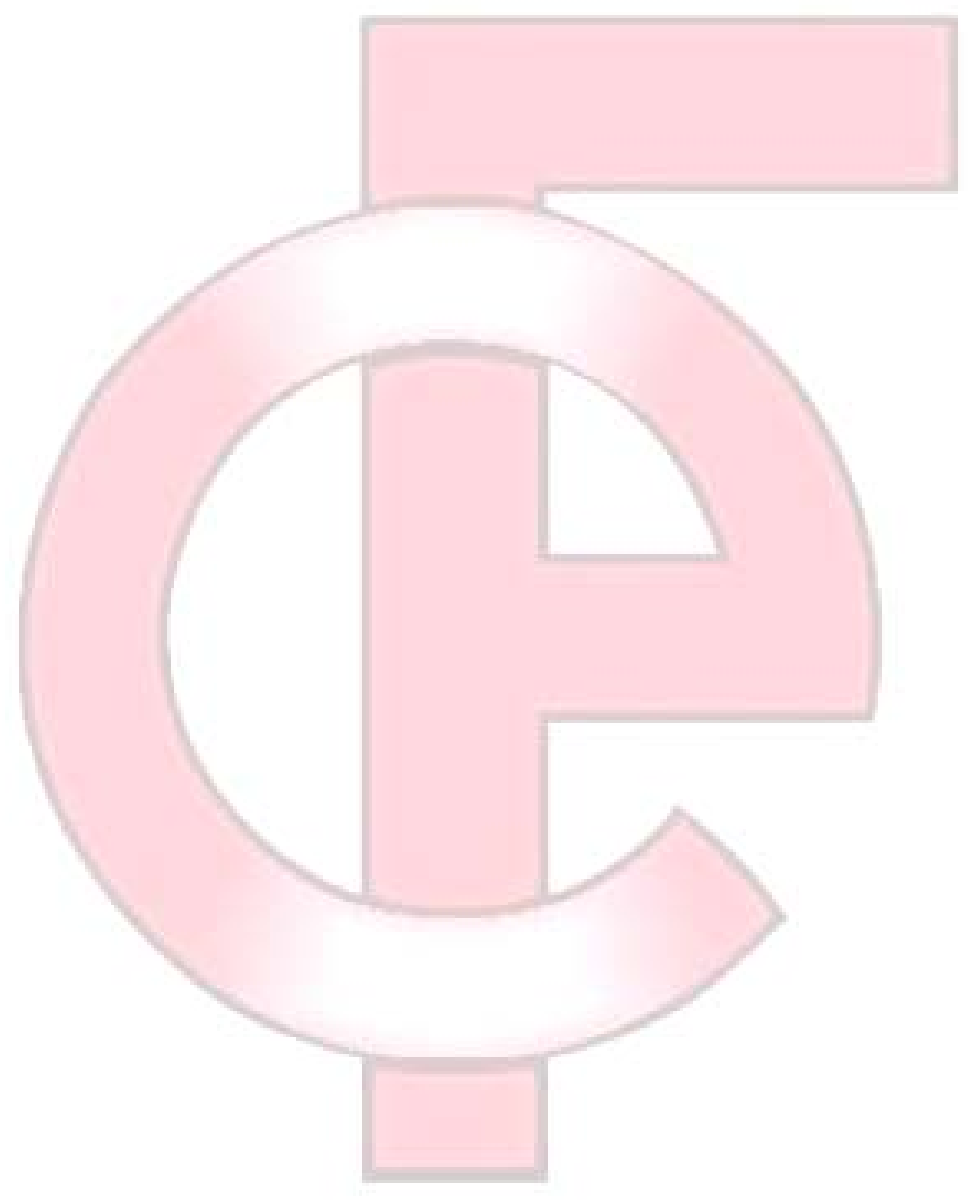

